auslaugen, das entstandene salzsaure Zink vom Gypse, Schwefelzink u. \&. w. trennen, mit Zinkplatten zur Abscheidung des fremden Metalls in Berührung lassen, filtriren und mit Aetzkalk zersetzen, wobei der salzsaure laalk zur neuen Arbeit wieder gewonnen würde, und dann in einem geeigneten Apparate reduciren und destilliren.

Auch $S$ a nd or fand, dafs weder die Destillation des gerreinen Zinks, noch die Behandlang desselben mit Schwefel und Talg ein reines Zink liefere, dos letzte Verfahren "überhaupt das schlechteste sey, und sich dabei auch viel Schwefelzinh bilde. Ein reines Oxyd läfst sich nach ihm ebenfalls nur durch Reduction des reinen Oxydes darstellen, und das reducirte Metall muls zweimal destillirt werden, da ea bei der ersten Destillation inumer kohlenhaltig ist. Dierelben Schlufsfolgen zieht $S \mathrm{ch}$ wati $\theta$ aus seinen Versuchen, so wie auch Laux und Fischer.

\title{
Ueber Bereitung des Calomels auf nassem Wege
}

\author{
von \\ Dr. Theodor Wilhelm Christian Martius, \\ Apothekerin Erlangen.
}

Die gebräuchlichste Methode, dieses Mercurialpräparat auf trochenem Wege zu bereiten, hat das Unangenehme, dafs der erhaltene feste Sublimat erst mühsan präparirt werden mufs, wobei eine Verunreinigung mit Staub, Kalls 4. 8. w. nicht zu rermeiuen ist. 
Um diesen Uebelständen zu begegnen, schlug Javel I) vor, den sublimirenden Calomel in hochendes Wasser zo leiten; Henry ${ }^{2}$ ) n̈nderte dies Nethode dahin $a b$, taf er suchte, die sublimirenden Calomeldïmpfe mit Wasserdỉmpfen in Berührung zu bringen ; allein $R \mathbf{a b}{ }^{3}$ ) zeigte, dars dieses Verfahren nicht so leicht gelingt, als man allgemein glaubt, was bei der Eigenthümlichkeit des versülsten Queck. sibbers, sich gern zn verdichten, und bei der Schwierigkeit zu sublimiren leicht zu erliüren ist. $\Lambda$ uch hat man gefürchtet, dafs der sublimirte Calomel öfters mit Aetz-Sublimat verunreinigt vorkommen künne, und ob dies gleichwoh! schwer zu glauben ist, so hat Baumé i) doch gezeigt, dafs es stattfindet. Deswegen soll man das feingeriebene Präparat zur Entfernung des Suhlimats mit Salınialiwasser kochen. Diese Reinigungs-Methode gab $P f$ af $f$ ') Veranlassung zu Versuchen, und er fand, dafs 200 Gran Salmiak, weno sio mit 100 Gran Calomel geliocht werden, 19 Gran des Letzteren auflüsen, eine Beobachlung, die frïher schon ron Pettenkofer 9) gemacht worden war, dessen Resultate wenig ron denen $P$ faffs abweichen, da er in einem ähnlichen Verhältnisse und auf dieselbe Art arbeitete wie dieser. Allein es ist nur za beachten, dafs wohl die wenigsten Apotheker den sublimirten feingeriebenen Calomel, kochend mit Salmiak, bebandeln, and dafs nie ein solehes Uebermars von Salmiak angewendet wird, um den Calomel seines etwaigen Sublimat-Gehaltes zo berauben, als $\mathrm{Pf}$ a ff angibt.

Ich habe mich recht oft einer kulten wässrigen Salmiak-

1) Brandes Archiv Band $V$ Seite 39.

2) Repertorium von Buchner Band XV Seite 15 .

3) Repertorium von Buchner Band XV Seite 118.

4) Hamburgisches Magazin Band XIV Seite 291.

5) Schweigger Journal Band XV Seite 100.

6) Hepertorium von Buchner Band III Seite 38. 
auflösung zur Reinigung cies (cingeriebenen sublimirten Calomeis bedicnt; allein mein Verfahren bestand darin, dafs ich den auf einem ausgespannten mit Fliefopapier belegten Colatorium belindlichen Calomel mit einer kalten filtrirten wässrigen Auflösung des Salmiaks übergofs. Gewöhnlich wurden auf 12 Uuzen Calomel 2 bis 3 Drachmen Salmiak genommen, die in 2 Pf. Wasser gelöset wurden. Die zuerst ablaufende Flïssigheit wurde zurückgegossen, nochmals aufgegeben, und dann der Calomel so lange mit destillirtem kaltem Wasser ausgewaschen, bis die Abwaschflüssigkeit Silbersalze nicht mehr trübte. Niemals konnte ich durch tines der geeigneten Reagentien in der ablaufenden. Flüssigkeit eine Spur von Sublimat entdecken. Angenommen auch, dafs ein Pfund in Arbeit genommener Calomel 2 Drachmen Aetz-Sublimat enthielte, so genügt die angeführte Menge Salmiaks hinlänglich, um diese schädliche und gefürchtete Beimischung zo entfernen. Dulk 7) verwirft das Auswaschen init Salmiakwasser ganz und gar, nichts desto weniger dïrfte es doch nicht so zweckwidrig seyn, den lialten $\Lambda$ bwaschwasser Salmiak zuzusetzen, wie Geigers) sagł, welcher fürchtet, dafs sich bei dieser Manipulation Sublimat erzeugt, was jedoch nur dann erfolgen wird, wenn die Auswaschung kochend geschieht. Bei der Auflösbarkeit des Alembrothsalzes dürfte jedoch dieses Verfahren auch ganz unnöthig seyn. Leicht mag es geschehen, dafs wenn man eine doppelte Menge Sulmiak zur kalten Auswaschung eines Theiles Calomels verwendet, allein grofs wird die Menge gewifs nie seyn.

Vor einigen Jahren hat $\mathrm{Osw}$ ald ${ }^{9}$ ), der seine Versuche in einem andern Verbïltnisse wie Pfaff anstellte, die Wabrheit der von mir gemachtus Beobachtungen bestätigt und

7) Pharınacopoea borussica Band II Seite 445 .

8) Geigers Handbuch der Pharmacie Zweite Auflage Seite 630.

9) Taschenhuch für Scheidekünstler 1828 Seite 24 . 
gefunden, dafs wenn 6o Theile Calomel und, Theil Salmiak einige Stunden lang gekocht werden, kaum bemerhbare Spuren von Sublimat in der abfiltrirten Flüssigkeit entdeckt werden konnten, und dafs kalt beide Substanden mit Wasser angerührt bei gewöinlicher Temperatur auf einander nicht wirken. Die Mühe des Präparirens und Scìlemmens bei dem sublimirten Calomel, wobei man selbst bei der gröfsten Vorsicht einer Verunreinigung mit Staub unỏ Kalk aus der Reibschaale nicht entgeht, ist auch wohl sehr in Betracht zu ziehen.

Das Fcinreiben des Calomels in Serpentinschaalen zu bewerkstelligen, was Oswald ${ }^{10}$ ) gethan hat, ist auf keinen Fall zu empfehlen, da bei der Weichheit des Materials eine sehr beträchtliche Verunreinigung stattinden mufs. Selbst in einer sehr dichten Marmorschaale präparirter Calomel zeigt Spuren von Kalk, wenn man das getrocknete Präparat mit Essigsäure digerirt und dann mit hleesaurem lkali reagirt.

Achat - oder Porphyr-Reibschalen sind wohl die zweckmälsigsten, allein kaum von der Grölse zu erhalten, um bedeutendere Menge sublimirten Calomels mit einem Male behandeln zu können. Deswegen verdient die von Scheele "), später von Buchbolz ") und vor wenigen Jahren von Geiger ${ }^{13}$ ) verbesserte Methode, den Calomel auf nassem Wege zu bereiten, wohl Beachtung, die bekanntlich darin besteht, dafs eine salpetersaure Quecbsilberoxydulauflösung mit Kochsalz niedergeschlagen wird. Neuerlichst empfieblt Kosthals 14) die Quechsilberaullösung mit salzsaurem Kalk

10) Taschenbuch für Scheidekünstler 1828 Seite 20.

11) Sclieele sämmtliche Schriften Band II Seite 163.

12) Trommsdorff Journal Band IX St. I Seite 65 und Band XIV. St. 2 Seite 120

13) Repertorium von Buchner Band XI Seite 58.

14) Meylinks Bibliothol Band V Seite 155. 
niederzapchlagen, was jedoch wohl nur in wissenschafticher Beziehung von lnteresse ist. Dull is) so wie auch O swald 16) sprechen davon, dals der auf nassem Wege bereitete Calomel eine heftigere Wirkung äufsere, als wenn er auf dem Präparirstein fein gerieben worden sey, ullein ich frage, welcher von den beiden angeführten Herren direkte Versuche anstellen liefs. Obschon auf mein bittliches Ver. langen eine bedeutende Menge Versuche mit aut nassem Wege bereitetem Calomel in dem hiesigen tlinischen Institute von dem Herrn Direhtor Professor D. J a eger angestellt wurden, so hat doch in leinem Fall dieses Präparat energischere oder kräftigere Wirkung gezeigt. Dies ist ein Factum, welches die Angaben der verehrten beiden Herren Collegen entkräftigen wird.

Ein sehr za berücksichtigender und allgemein empfohlener Umstand bei Bereitung des Calomels auf nassem Wege ist, dafs eine blos salpetersaure quechsilberoxydulhaltige $\Delta$ uflösung zur Niederscblagang verwendet wird 1i), um einer Verunreinigung mit basisch salpetersaurem Quechsilbercxyde zu begegnen, die sich jedoch nach Bucholz ${ }^{18}$ ) nur bei sehr unachtsamer Manipulation ereignen wird.

Die Bereitang einer salchen reinen salpetersauren Quecksilberoxydullösung im Grofsen ist immer mit einigen Schwierigkeiten verknüptt, und ich bediene mich schon seit einigen Jahren eines Verfahrens den Calomel auf nassem Wege zo bereiten, weiches ich der Prüfung meiner geehrten Herren Collegen unterwerfe.

Es besteht in Folgendem:

Eine gewöhnliche salpetersaure Quecksilberauflösung,

15) Pharmacopoea borusaica Band II Seite 446.

16) Taschenbuch für Seheidekünstler 1828 Seite 29.

17) Repertorium ron Buchner Baud XI Seite 72.

18) Taschenbuch für Scheidekünstler 1807 Seite 75. 
welche ich entweder dadurch bereite, dafs ich viel Quecksilber mit verdünnter Salpetersäure übergiefse zum Kochen erhitze, oder dafs ich viel Queclisilber mit verdünnter Salpetersäure hoche und unter öfterm Umschütteln einige Monate lang über einem Ueberschufs von metalliscbem Quecksilber stehen lasse. Eine solche salpetersaure Quechsilberauflösung rerdünne ich mit viel destillirtem Wasser, wobei nie etwas basisch salpetersaures Quecksilber ausgeschieden wurde, filtrire und schlage sie nun nicht mit Kochsalz, sondern mit einer filtrirten wässrigen Salmiakaullösung nieder. Es entsteht ouf der Stelle ein sehr häufiger weifser Niederschlag, doch wird Salmiahlösung im Ueberschufs zugesetzt, und anf schickliche Weise ausgewaschen; nach dem Trocknen wird ganz vortrefflicher höchst fein vertheilter Calomel erhalten. Einige Male beobachtete ich das Freiwerden von calpetriger Stiure, welche durch Geruch und Farbe zu erkennen war; es trat dies vorzüglich dann ein, wenn die salpetersaure Quecksilberlösung stark sauer war and viel Quech. silheroxydulsalz enthielt. Achnliches beobachtete auch $\mathbf{B u}$ cholz ${ }^{19}$ ).

Aus der crst abgelaufenen Flüssigheit hann durch Zusatz von kohlensaurem Natron noch weifscr Prücipitat niedergeschlagen werden, weil in beiden oben angegebenen Fällen die Quechilberlösung stets mehr oder weniger salpetersaures Quecksilberoxyd enthält. War die Quectsilberlö. sung sauer, so war die Ausbeute an weifsem Präcipitat stets gröfser, indem sich in diesem Falle mehr salzsaures Quechsilberoxydammoniak gebildet hatte. Das salpetersaure Quecksilberoxyd gibt mit Salmialı eine sehr leicht lösliche Trippelverbindung, und deswegen wird auch der auf diese Art iereitete Calomel nie mit basisch-salpetersaurem Mercuroxyd verunreinigt seyn.

19) Trommsdorff Journal Band XXI St. 2 Seite 107. 
Aus dem Angeführten ist ersichtlich, dafs diese Methode Vorzüge ror allen bis jetzt bethannten Bereilungsarteil des Calomels anf nassem Wegp hat, die darin bestehen,

1) dafs es ganz gleichgültig ist, ob die zur Niederschlagung bestimmte salpetersaare Qucchsilberanflösung vicl oder wenig Quccksilberoxydulsalz enthält, da auf der größsern oder geringern Menge des darin befindlichen Oxyculsalzes nur die gröfsere oder geringere Ausbeute an Calomel beruht;

2) dafs dadurch die Darstellung einer reinen salpetersauren Quecksilberoxydulauflüsung unnöthig wird;

3) dafs aus der dabei gewonnenen Abwaschflüssigheit durch Zusatz von Hoblensaurem Natron oder Ilali weifser Präcipitat gewonnen werden kann;

4) dafs das erhaltene Präparat frei von jeder Verunreinigung ist, welcher der darch Präpariren bereitete Calomel stets unter worfen seyn wird, und dafe

5) das erhaltene Produht in Bezag auf Reinheit, Schön. heit und Güre ouch gar nichts zu wänschen übrig läfst. Schon im Jahre 1825 habe ich mit einem meiner fleifsigwen Schüler, Herrn Apotheker $S a u b e r$ in Rothenburg an der Tauber, das auf die ron mir beschriebene Art gewonnene Produkt vielfach untersucht, allein anf keine Weise konnte nur die geringste Spur irgend einer Verunreinigung entdeckt werden; chemisch war ebenfalls kein Unterschied zu bemerken, nur mufs das Präparat gut, and mit kaltem Wasser lange ausgewaschen werden, ein zu beherzigender Umstand. Anführen mufs ich noch, dafs der nach meiner Angabe a..s cinex concentrirten Autlösung bereirete Calomel einmal ein festes bröckliches Anseben hatte, auch getrocknet etwas soliwerer zu reiben war, ohne jedoch durch starkes Pressin nach Stehle ${ }^{29}$ dem durch Sublimation exhaltenen Calomel ähnlich zu werden.

20) Magaxin für Pharmacie Bad XV XVII Seite $5 y$ 
Uebrigens ist früher schon von Geige ${ }^{21}$ ), dann von Trautwein 2i), Guibourt $\left.{ }^{2}\right)$, Zaboada, Vogel, Gruner und ncuerlichst von $S$ chweinsberg ${ }^{24}$ ) die Identität Je: unf nassem und trocknem Wege bereiteten Colomels dagethan worden, so dafs wohl hein Zweifel über die gleichartige chemische Constitution beider Produkte mehr stattinden lann.

Was die medicinische Anwendung des auf die angegebene Weise gewonnenen Prïparates anbelangt, so beweisen die früher angeführten Versuche zur Genïge, dals seine Wirhsamlieit der des präparirten durch Sublimation gewonnenen Calomels nicht nachsteht, so wie auch bei einer nur etwas sorgfältigen Arbeit eine gefährliche Beimischung nicht stattfinden kann. Im Gegentheil ist pher von dem durch Sublimation und durcb Feinreiben bersiteten Calomel eine unsichere Wirkung zu vermuthen, da das Prapariren ja ganz in der Willuühr des A pothekers steht. Auf dieselbe Weise äu(sert sich auch Jung ${ }^{25}$ ), der bei dem Gebrauch des auf nassem Wege bereiteten Calomels nie eine schlimme Wirlung beobachtete und am angeführten Ort sein Verfabren Leschreibt; er läfst mit Hochsalzlösung niederschlagen. Ueberhaupt haben wir ja hein Bestimmungsmittel für die Feinheit eines Pulvers; dem einen scheint etwas fein, was der andere noch nicht dafür erkennt, und da wir keine Gränze Lennen, in dem gegebenen Falle die Feinheit des präparir. ten Calomels zu messen, so dürfte die Anwendung des durch Niederschlagung gewonnenen Calomels eher einen Vorzag haben, weil das Präparat stets dieselbe Feinheit zeigen wird.

21) Repertorium von Buchner Band XI Seite 76 .

22) * $*$ V XII * 158.

23) Trommsdorfi $N$. Journal Band XX St. , Seite 230.

24) Magazin für Pharmacie Band XXVII Seite 241.

s) Trommsdorfi Feues Journal Band XXII Stüch , Seite 62 . 
Durch Schlemmen hönnte präparirter Calomel wohl höchst fein erhalten werden, jedoch hat bis jetzt noch keine Pharmacopöe dies zu thun befohlen.

Es würde mich sehr freuen, wenn mehrere meiner Herreu Collegen diese Methode einer genauern Prüfung anterwerfen und ihre Risultate in dieser Zeitschrift mittheilen würden. Herr College Trautwein in Nürnberg, der gegen mich äufserte, dafs der so gewonnene Calomel wohl eino Trippelverbindung mit Ammoniak seyn könnte, hat bei sorgfältiger Untersuchung jedoch keine Spur des fraglichen Stoffes entdecken können.

Schliefslich möchten hier noch einige Versuche ein Plätzchen finden, die ich mit Calomel auf nassem und trocknem Wege bereitet, so wie mit Sublimat anstellte und die einige merkwürdige Erscheinungen zeigen. Guibourt 26) hat schon bemerlit, dafs niedergeschlagener Calomel, wenn man ihn in einer Glasröhre sublimirt, wenig das Lakmus röthenden Dunst gibt; ich fand, dafs wenn ein Stüchichen Jurch Sublimation erhaltener Calomel aufs Neue in einem Glasröhuchen gegeben wird, der aufsteigende Sublimat das Lakmuspapicr röthet; dasselbe geschieht, wean das Stückchen schon geglüht ist, und auch dann wenn die Röhre, in welcher die Sublimation erfolgt, rorher stark erwärmt. ist.

Sublimat verhält sich ebenso.

Die Ursache, warum bei dem auf nassem Wege erhaltenen Calomel die Erscheinung Talinuspapier zu röthen schneller eintuit,, rüht wohl daher, dafs der fein vertheilte Calomel weit leichter and früher sublimirt, als der in Stükken befindliche durch Sublimation gewonnene; vielleicht entbält der erstere auch etwas hygrometrisches $W$ asser, welches die Erscheinung beg:"nstigt. Es scheint hier eine theilweise Zersetzung der beiden Chloride stattzufinden; atcel:

20) Trommsdos if Neues Joarnal Band XX Stück Seite 225. 


\section{6}

wärc es möglich, dafs wenig beigtmischte organische Stoffe etwas Calomel zerlegen, rodurch Salzsäure frei wird, während das reducirte Quecksilber den Sublimat mehr oder weniger färht. Vielleicbt könnte auch die geringe Menge Feuchtigkeit der Atmosphäre eine Zerlegung bewirben.

\section{$=$ \\ Ueber Jod äther \\ von}

James F. W. Johnston.

(The Lond. and Edinb. philos. Magaz. and Jomn. of Science. 1833. 1I. 415.)

Wenn man in heifse Salpetersäure, die in eizer geräumigen Flasche sich befindet, eine gesättigte Auflösung ron Jod in Alliohol bringt, so findet eine heftige Wirkung statt, mit. Entwichlung von Salpeteräther, Essigsäure, und Salpo tergas, and die Farbe der Jodauflösung verschwindet. Wena die Hitze nachläfst und so lange fastes Jod zugesetzt wird als die Wirkung noch stattfindet und die Farbe versehwindet, so setzt sich beim Erhalten eine durchscheinende gelbliche ölähnliche Flüssigheit ab, die schwerer ist als Wasser und folgende Eigenschatten zeigt;

1. Sie hat einen starken darchdringenden Geruch, der sehr verschieden ist ron dem des Hydriodäthers nach GayLussac und einen anhaltenden brennenden Geschmack.

2. Wenn sie frei ron Jodüberschufs ist, hat sie eine sehr blasse gelbe Farbe; eine geringe Erwärmung reicht jedoch hin sie zu entfärben, wobci eine theilweise Zersetzang entsteht.

3. Sie ist nicht leicht entzündlich und lann nicht ohne Zersetzung verlliehtigt werden. Durch das Licht und die 